\title{
BANDWIDTH ASSESSMENT FOR MULTIROTOR UAVs
}

\author{
Gastone FERRARESE* \\ *Faculty of Engineering, Department of Industrial Engineering, University of Bologna, Via Risorgimento 2, 40136, Bologna, Italy \\ gastone.ferrarese2@unibo.it
}

received 14 July 2016, revised 29 May 2017, accepted 31 May 2017

\begin{abstract}
This paper is a technical note about the theoretical evaluation of the bandwidth of multirotor helicopters. Starting from a mathematical linear model of the dynamics of a multirotor aircraft, the transfer functions of the state variables that deeply affect the stability characteristics of the aircraft are obtained. From these transfer functions, the frequency response analysis of the system is effected. After this analysis, the bandwidth of the system is defined. This result is immediately utilized for the design of discrete PID controllers for hovering flight stabilization. Numeric simulations are shown to demonstrate that the knowledge of the bandwidth is a valid aid in the design of flight control systems of these machines.
\end{abstract}

Key words: Multirotor, PID, Attitude Stabilization, Flight Control, Bandwidth

\section{INTRODUCTION}

Multirotor helicopters are Vertical Take Off and Landing (VTOL) flying vehicles that have gained, in the very last years, the attention of both academic research institutions and commercial companies. As flying systems, in the majority of cases, they feature a cluster of propellers, driven by electric motors, that permit them to be lifted and piloted in a perfectly balanced manner (Mahony et al., 2012; Stepaniak, 2009).

Applications of these machines regard surveillance, environmental monitoring, also in places where the human presence can not be admitted, and ludic activities.

Beside that, worldwide academic centers have developed various projects concerning the design of these aircrafts and the improvement of their performances. One branch of the research deals obviously with the problem of flight stabilization, that, for rotorcraft, represents a troublesome concern (Padfield, 1996, Pounds et al. 2009; Du, 2015; Raffo et al., 2009; Crowther, 2011).

Digital controllers are necessary for multirotor aircraft, to be controlled by an on--board digital microcontroller. Thus, the choice of parameters as the sampling time of a discrete PID must be properly addressed (McLean, 1990).

Multirotor aircrafts are generally provided with linear PID regulators for attitude and flight stabilization (Sa and Corke, 2011; Koszewnik, 2014). This type of controllers is utilized for the relative ease of implementation. Their tuning can be accomplished with "trial--and--error" attempts even by people without any notion of control theory, as in the case of commercially available systems (PixHawk, Ardupilot,etc.).

This approach however cannot represent an acceptable way to control systems synthesis, by a scientific and also engineering point of view.

In closed-loop control system design for rotorcraft, and also for other dynamic systems, a key concept is that of "bandwidth", as Padfield (1996) clearly stated in his capital book. Unfortunately, a rigorous study of this parameter for multi--rotor aircraft is not yet available in literature.

This technical note is the first attempt to fill this void. An analytic method to find the bandwidth of a multirotor helicopter is described. This result is based on the study of the frequency responses of this type of aircraft, derived from the analytic linear modelling of dynamics (Ferrarese, 2017).

The result is then utilized for the sizing of digital controllers for attitude stabilization. This is to show that the theoretic frequency analysis can represent a valid tool in the design and improvement of the performances of this kind of aircraft, particularly in the choice of Off-the-Shelf (OTS) components for control systems.

The argument of the present note is based on the theoretical results described in previous works of the author (Ferrarese, 2015; 2016; 2017).

\section{DYNAMIC MODELLING}

The linear dynamic modelling of a generic aircraft is defined by the following vector equation (McLean, 1990; Padfield, 1996).

$\dot{\mathbf{x}}=\mathbf{A} \mathbf{x}+\mathbf{B} \mathbf{u}$

$\mathbf{x}$ is the state vector of the system, $\mathbf{u}$ the control vector, $\mathbf{A}$ the stability matrix and $\mathbf{B}$ the control matrix. To this differential system the initial reference condition must be added. For a multirotor helicopter, the hovering flight condition represent an effective choice (Crowther et al., 2011).

$\mathbf{x}$ contains the perturbations of the attitude $(\varphi, \theta, \psi)$, of the linear velocities $(u, v, w)$ and of the angular rate variables $(p, q, r)$

$\mathbf{x}=[\varphi, \theta, \psi, u, v, w, p, q, r]^{T}$ 
$\mathbf{u}$ contains the small variations of the 4 control inputs. Any of them acts as a changing of the speed rates of the rotors, according to a precise maneuver, and are here defined in analogy with the controls of a traditional helicopter (Padfiled, 1996).

$\mathbf{u}=\left[u_{\text {col }}, u_{\text {lon }}, u_{\text {lat }}, u_{\text {rud }}\right]^{T}$

$u_{c o l}$ imposes to the aircraft a vertical variation of velocity, if starting from a hovering flight condition. $u_{\text {lon }}$ and $u_{\text {lat }}$ instead direct the flight in the horizontal plane and the relative changes in attitude. $u_{r u d}$ instead commands the yaw rate variations.

$\mathbf{A}$ is the state matrix and contains all the stability derivatives. These derivatives define the effect of the state vector on the state vector dynamics itself. $B$ instead defines the effect of input variations of the state dynamics. $\mathbf{A}$ is a $9 \times 9$ matrix and $\mathbf{B}$ a $9 \times$ 4 matrix.

However, aiming at the analysis of flight dynamic stability, the study can be limited to a reduced order model (Ferrarese, 2017). This reduced dynamic system is defined by the following set of equations, that contains only the dynamics of the horizontal motion variables.

$\left[\begin{array}{c}\dot{\theta} \\ \dot{u} \\ \dot{q}\end{array}\right]=\left[\begin{array}{ccc}0 & 0 & 1 \\ -g & X_{u} & X_{q} \\ 0 & M_{u} & M_{q}\end{array}\right]\left[\begin{array}{l}\theta \\ u \\ q\end{array}\right]+\left[\begin{array}{c}0 \\ 0 \\ M_{l o n}\end{array}\right] u_{l o n}$

where: $g$ - gravity acceleration; $X_{u}$ and $X_{q}$ - the stability derivatives that define the effect of $q$ and $u$ on the translational dynamics along the $x$ body - axis (longitudinal axis) of the aircraft; $M_{u}$ and $M_{q}$ - the stability derivatives that define the effect of $q$ and $u$ on the rotational dynamics about the $y$ body_axis (lateral axis) of the aircraft; $M_{l o n}$ - the control derivative that define the effect of a longitudinal input $u_{\text {Ion }}$ on the angular dynamics about the same $y$ body-axis.

The last linear dynamic system can be shown to be characterized by high frequency real stable pole equal to $M_{q}$, and two low frequency poles that can be complex conjugate or both real, with one of them unstable. The design of the stabilization system must evidently cope with the presence of these unstable poles.

\section{FREQUENCY RESPONSE ANALYSIS}

From Eq. (4) the transfer functions of the $(\theta, u, q)$ can be extracted (Ferrarese, 2016). Numerical evaluations show that the values of $X_{u}$ and $X_{q}$ are negligible with respect to the other derivatives. Then, these two last derivatives can be omitted in the transfer functions calculation.

$$
\left[\begin{array}{l}
G_{\theta} \\
G_{u} \\
G_{q}
\end{array}\right]=\left[\begin{array}{l}
\frac{M_{l o n} s}{s^{3}-M_{q} s^{2}+g M_{u}} \\
\frac{-g M_{l o n}}{s^{3}-M_{q} s^{2}+g M_{u}} \\
\frac{M_{l o n} s^{2}}{s^{3}-M_{q} s^{2}+g M_{u}}
\end{array}\right]
$$

Now, the frequency response can be analyzed with the Bode diagrams of the functions of Eqq. (5). In Fig. (1) the frequency response of the pitch rate $q$ is shown. The data for the reference aircraft are given in Tab. (1).

From the Bode plot interesting informations can be immediately pointed out. There is a peak, that announces a probable resonance behavior, at a frequency near to that of the unstable complex poles. In the proximity of this same peak the phase diagram almost reaches the value of $180^{\circ}$. These two facts can bring to affirm that at those low frequencies some oscillatory and unstable behavior of the system could manifest.

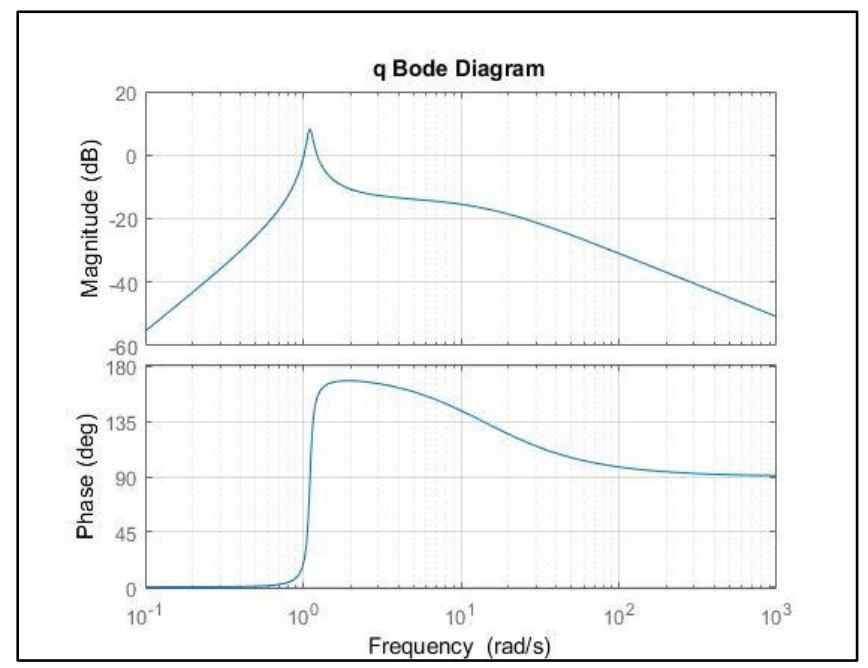

Fig. 1. Bode diagrams of $G_{q}(s)$

Tab. 1. Data of Reference Aircraft

\begin{tabular}{|c|c|c|}
\hline Quantity & Value & Unity \\
\hline$\rho$ & 1.225 & $\mathrm{~kg} \mathrm{~m}^{-3}$ \\
\hline$g$ & 9.81 & $\mathrm{~m} \mathrm{~s}^{-2}$ \\
\hline$I_{y y}$ & 0.05 & $\mathrm{~kg} \mathrm{~m}^{2}$ \\
\hline$N_{\text {rot }}$ & 6 & \\
\hline$h$ & -0.1 & $\mathrm{~m}$ \\
\hline$R$ & 0.2 & $\mathrm{~m}$ \\
\hline$m$ & 4 & $\mathrm{~kg}$ \\
\hline$\Gamma_{j}$ & 5 & $\circ$ \\
\hline$\xi_{j}$ & 5 & $\circ$ \\
\hline
\end{tabular}

At a frequency about that of the stable real pole $\left|M_{q}\right|$ the gain starts to rapidly decrease.

This result signifies that the bandwidth of the multirotor helicopter under exam can be assuredly put around the $\left|M_{q}\right|$ frequency.

$\omega_{b w}=\left|M_{q}\right|$

\section{CONTROL SYSTEM DESIGN}

In this section an example of the application of the bandwidth to flight stabilization systems design is illustrated.

A digital PID controller is defined by the following equation (Magnani et al., 2007).

$G_{c}(z)=\mathrm{K}_{P}+K_{I} \frac{T_{S} z}{z-1}+\mathrm{K}_{D} \frac{z-1}{T_{S} z}$

The three gains $K_{P}, K_{I}$ and $K_{D}$ can be taken from the relative analog regulators, that can be sized through the Routh criterion. In the present case, limiting the analysis to the system of Eq. (4), two feedback actions can be included. One refers to the attitude $\theta$. The other to the velocity $u$. Two PD controllers represent a solution that can grant the stability of the system (Ferrarese, 2016). 
$\left\{\begin{array}{l}K_{P, \theta}=-K_{1} \\ K_{D, \theta}=\frac{K_{1}}{M_{q}} K_{2}\end{array}\right.$

$\left\{\begin{array}{c}K_{P, u}=K_{1} \\ K_{D, u}=-\frac{K_{1}}{M_{q}} K_{2}\end{array}\right.$

$K_{2}$ must be greater than 1 and

$K_{1}=\frac{M_{q}}{M_{l o n}}$.

To complete the design of the discrete controller it is necessary to choose a proper value for the sampling time $T_{s}$.

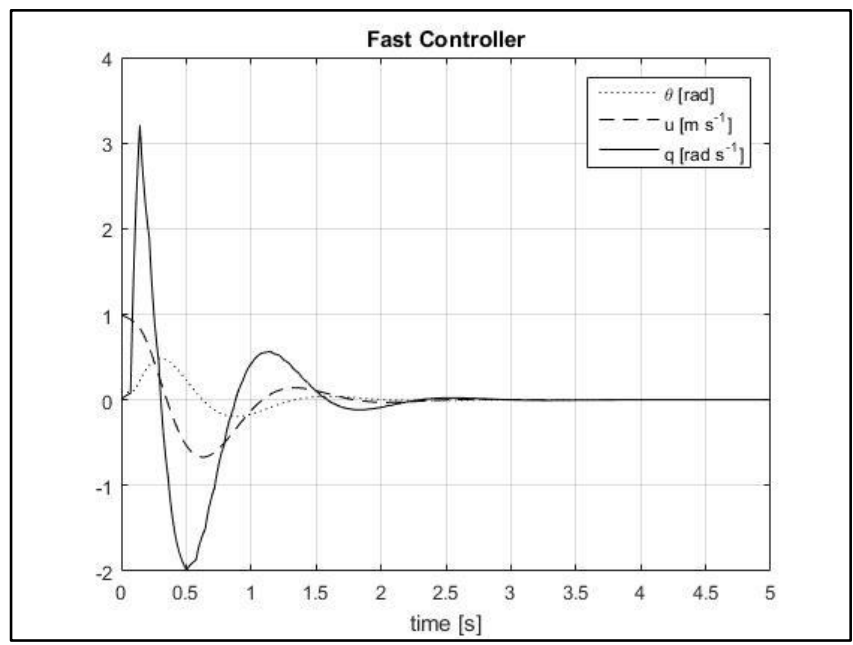

Fig. 2. High Frequency PID Response

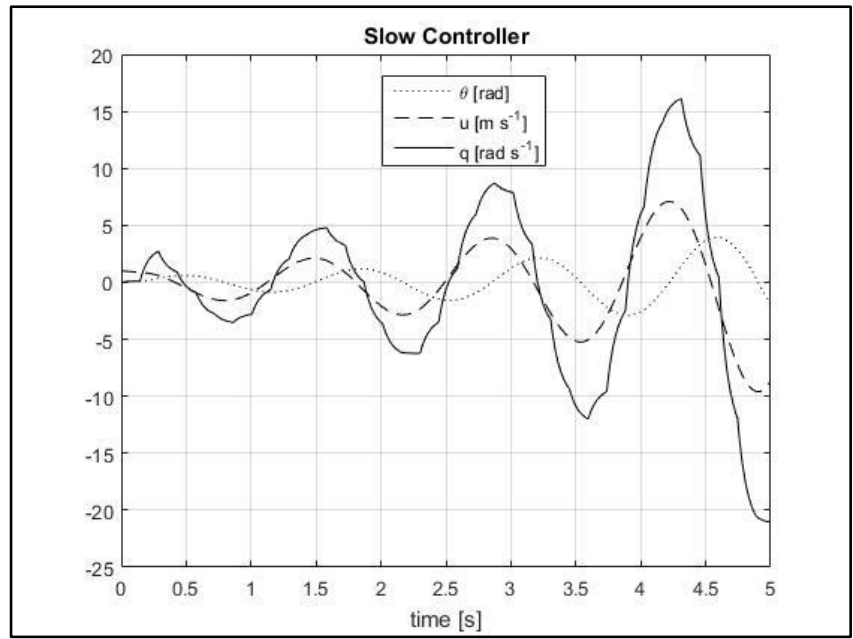

Fig. 3. Low frequency PID Response

The sampling time is a parameter linked to the performance of the sensor and electronic system of the aircraft. Extremely low sampling time (or very high sampling frequency time) could represent an excessive burden for the control unit of the aircraft. Thus, to assess the minimum acceptable value for $T_{s}$ can have an important impact on the design of the machine itself and, above all, of its control systems. This result can push towards an optimal choice of the sensors and computers to be installed on the aircraft and towards the optimization of the cost and performances of the entire machine.
Having previously defined the bandwidth $\omega_{b w}$ of the system, it is reasonable to assume the value of $T_{s}$ equal to the reciprocal of $\omega_{b w}$.

This choice can be tested through simulation. Imposing an initial disturbance from the hovering condition to the system of Eq. 4, the effect of the two discrete PID can be evaluated. The first PID runs with a sampling time equal to $1 /\left|M_{q}\right|$. The second $P I D$ runs at a frequency equal to the half of the bandwidth. The results of simulations are depicted in Fig. 2 and Fig. 3. In Fig. 3 the oscillatory behavior predicted from the Bode diagram of Fig. 1 can be easily witnessed. The value of $K_{2}$ has been put equal to 5 .

\section{CONCLUSION}

In this paper the theoretical analysis of the bandwidth of multirotor helicopters is accomplished, for the first time in a scientific publication. A set of mathematical tools for the study of the flight stability of this type of aircraft is provided. The equations are utilized to the characterization of their frequency response and, then, to the description of unstable behaviors. This same analysis has brought to the definition of the bandwidth of these dynamic systems. This parameter provides notable informations about the stabilizing actions to be imparted to the aircraft. The result is immediately applied to the design of stabilization systems.

\section{APPENDIX}

The stability and control derivatives of Eq. (4) can be retrieved from Ferrarese $(2015,2017)$. In those works all the necessary explanations and theoretical demonstrations can be found.

Here those utilized for the simulation are repeated:

$$
\begin{gathered}
M_{u}=-\frac{1}{I_{y y}} \sum_{j}^{N_{\text {rot }}} \frac{\partial C_{T}}{\partial \mu_{z}} \rho A \Omega_{0} R \mathbf{T}_{\boldsymbol{j}}(3,1)\left[h \mathbf{T}_{\boldsymbol{j}}(3,1)\right. \\
\left.+\left(-l \cos \left(\delta_{j}\right) \mathbf{T}_{\boldsymbol{j}}(3,1)\right)\right] \\
M_{q}=-\frac{1}{I_{y y}} \sum_{j}^{N_{\text {rot }}} \frac{\partial C_{T}}{\partial \mu_{z}} \rho A \Omega_{0} R\left[(-h) \mathbf{T}_{\boldsymbol{j}}(3,1)\right. \\
\left.+\left(l \cos \left(\delta_{j}\right) \mathbf{T}_{\boldsymbol{j}}(3,3)\right)\right]^{2}
\end{gathered}
$$

$M_{\text {lon }}$

$=-\frac{1}{I_{y y}} \sum_{j}^{N_{\text {rot }}} \frac{m g}{N_{\text {rot }} \cos \left(\Gamma_{j}\right) \cos \left(\xi_{j}\right)} \frac{2}{\Omega_{0}}$.

$\cdot\left[-\mathbf{T}_{\boldsymbol{j}}(3,3)(-l) \cos \left(\delta_{j}\right) \operatorname{sign}\left\{\delta_{j}\right\}\right.$

$\left.-\mathbf{T}_{\mathbf{j}}(3,1) h \operatorname{sign}\left\{\delta_{j}\right\}\right]$

where: $m$ - the mass of the aircraft, $I_{y y}$ - the inertia moment around the $y$ body axis of the aircraft, $C_{T}$ - the rotor thrust coefficient, $\mu_{z}$ - the climb ratio of the aircraft, $R$ - the rotor radius, $A$ the rotor disk area, $\Omega_{0}$ - the rotor hovering spin rate of the rotors, $N_{\text {rot }}$ - the number of rotors of the aircraft, $\left(\delta_{j}, \Gamma_{j}, \xi_{j}\right)$ - a triplet of angles, named azimuth, dihedral and tilt, that defines the orientation of the $j$-th rotor, $\mathbf{T}_{j}$ - the relative rotation matrix, $h$ and $I$ - the distances of the rotor disk centers form the center of gravity of the aircraft, respectively the first parallel to the $z$ vertical body axis of the aircraft and the second perpendicular to the same axis.

The data for simulation are presented in Tab. (1). 


\section{REFERENCES}

1. Crowther B., Lanzon A., Maya-Gonzalez M., Langkamp D. (2011), Kinematic Analysis and Control Design for a Nonplanar, Journal of Guidance Control and Dynamics, 34(4):1157-1171.

2. Das A., Lewis K. S. (2009), Dynamic Inversion with Zero-Dynamics Stabilisation for Quadrotor Control, IEEE Proceedings Control Theory and Application, 3(3), 303-314.

3. Du G., Quan Q., Yang B., Cai K. (2015), Controllability Analysis for Multirotor Helicopter Rotor Degradation and Failure, Journal of Guidance Control and Dynamics, 38(5): 978-984.

4. Ferrarese G. (2015), Dynamics and Control Issues of Multi-Rotor Platforms, PhD Thesis, University of Bologna, Italy.

5. Ferrarese G. (2016), PID Augmented Stability of Multi-Rotor Helicopters, MAYFEB Journal of Control and Systems Engineering, Vol. 1,1-22.

6. Ferrarese G. (2017), Natural Motion of a Multi-Rotor Aircraft, Proceedings of the Institution of Mechanical Engineers, Part G: Journal of Aerospace Engineering (SAGE Journals), Research Article First Published online: January 5, 2017.

7. Koszewnik A. (2014), The Parrot UAV Controlled by PID Controllers, Acta Mechanica et Automatica, 8(2), 65-69.

8. Magnani G., Ferretti G. and Rocco P. (2007) Tecnologie dei sistemi di controllo, 2nd Ed, McGraw-Hill Italia.

9. Mahony R., Kumar V., Corke P. (2012), Multirotor Aerial Vehicles: Modeling, Estimation, and Control of Quadrotor, IEEE, Robotics \& Automation Magazine, 10(3), 20 - 32.
10. McLean D. (1990), Automatic Flight Control Systems, Prentice Hall.

11. Padfield G.D. (1996), Helicopter Flight Dynamics, $2^{\text {nd }}$ Ed. Blackwell Science Ltd.

12. Pounds P., Mahony R. and Corke P. (2010), Modelling and Control of a Large Quadrotor Robot, Control Engineering Practice, 18(7), 691-699.

13. Raffo G.V., Ortega M. G., Rubio F. R. (2009), An integral predictive/nonlinear $\mathrm{H}$ infinity control structure for a quadrotor helicopter, Automatica, 46, 29-39.

14. Sa I., Corke P. (2011) Estimation and Control for an Open-Source Quadcopter, Proceedings of Australasian Conference on Robotics and Automation, Monash University, Melbourne, Australia.

15. Stepaniak M.J., van Graas F., de Haag M.U. (2009), Design of an Electric Propulsion System for a Quadrotor Unmanned Aerial Vehicle, Journal of Aircraft, 46(3), 1050-1058. 